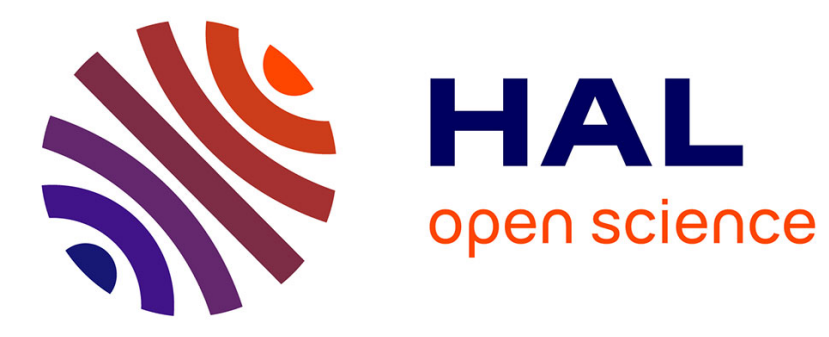

\title{
Surface effects on exciton diffusion in non polar $\mathrm{ZnO} / \mathrm{ZnMgO}$ heterostructures
}

Georges Sakr, Corinne Sartel, Vincent Sallet, Alain Lusson, Gilles Patriarche,

Pierre Galtier, Julien Barjon

\section{- To cite this version:}

Georges Sakr, Corinne Sartel, Vincent Sallet, Alain Lusson, Gilles Patriarche, et al.. Surface effects on exciton diffusion in non polar $\mathrm{ZnO} / \mathrm{ZnMgO}$ heterostructures. Journal of Physics: Condensed Matter, 2017, 29 (48), pp.485706. 10.1088/1361-648X/aa8a03 . hal-02972151

\section{HAL Id: hal-02972151 \\ https://hal.science/hal-02972151}

Submitted on 6 Dec 2021

HAL is a multi-disciplinary open access archive for the deposit and dissemination of scientific research documents, whether they are published or not. The documents may come from teaching and research institutions in France or abroad, or from public or private research centers.
L'archive ouverte pluridisciplinaire HAL, est destinée au dépôt et à la diffusion de documents scientifiques de niveau recherche, publiés ou non, émanant des établissements d'enseignement et de recherche français ou étrangers, des laboratoires publics ou privés.

\section{(ㄷ)(1) $\$$}

Distributed under a Creative Commons Attribution - NonCommercial| 4.0 International 


\title{
Surface effects on exciton diffusion in non polar $\mathrm{ZnO/ZnMgO}$ heterostructures
}

\author{
G Sakr ${ }^{1}$, C Sartel ${ }^{1}$, V Sallet ${ }^{1}$, A Lusson ${ }^{1}$, G Patriarche ${ }^{2}$, P Galtier ${ }^{1}$ and J Barjon ${ }^{1}$ \\ 1 GEMaC, Université de Versailles St Quentin en Yvelines, CNRS, Université Paris-Saclay, \\ 45 avenue des Etats-Unis, 78035 Versailles Cedex, France \\ 2 LPN CNRS, UPR20, 91460 Marcoussis, France
}

The diffusion of excitons injected in $\mathrm{ZnO} / \mathrm{Zn}_{0.92} \mathrm{Mg}_{0.08} \mathrm{O}$ quantum well heterostructures grown by metal-organic-vaporphase-epitaxy on non-polar $\mathrm{ZnO}$ substrates is investigated at room temperature. Cathodoluminescence linescans in a fieldemission-gun scanning-electron-microscope are performed across cleaved cross-sections. A $55 \mathrm{~nm}$ diffusion length is assessed for excitons in bulk $\mathrm{ZnMgO}$. When prepared as small angle bevels using focused ion beam (FIB), the effective diffusion length of excitons is shown to decrease down to $8 \mathrm{~nm}$ in the thinner part of the slab. This effect is attributed to non-radiative surface recombinations, with a $7 \times 10^{4} \mathrm{~cm} \mathrm{~s}^{-1}$ recombination velocity estimated at the FIB-machined $\mathrm{ZnMgO}$ surface. The strong reduction of the diffusion extent in such thin lamellae usually used for transmission electron microscopy could be use improve the spatial resolution of cathodoluminescence images, often limited by diffusion processes.

Keywords: excitons, diffusion, heterostructures, cathodoluminescence

\section{Introduction}

Diffusion lengths of excess charge carriers are key parameters which monitor semiconductor devices under injection. They directly impact the performances of optoelectronic devicesdedicated to light emission (LEDs, lasers) or absorption (photodetectors, solar panels). The diffusion properties of the injected excess charge carriers obviously relies on (i) the operating temperature (ii) the intrinsic properties of the material (iii) the density and nature of the defects it contains depending on the growth method used, but also (iv) on surface effects which may become dominant when nanosized objects are involved. From a characterization point of view, the diffusion properties of excess carriers are also a strong limitation for the spatial resolution of cathodoluminescence images: the spatial extent of charge carriers caused by diffusion is most often much larger than the probe beam size. More generally, it is clear that there is a need for nondestructive characterization tools able to measure diffusion lengths on real objects with a suitable accuracy.

The diffusion length is given by $L=\sqrt{D \tau}$, where $D$ is the diffusivity and $\tau$ the lifetime of carriers injected in excess. The range of carrier diffusion lengths in bulk semiconductors is extremely wide: it covers the millimeter to the nanometer scale, depending on the crystal quality and purity. In indirect semiconductors, large carrier lifetimes are generally responsible for long-range diffusion (e.g. up to $1.2 \mathrm{~mm}$ in pure silicon (Saritas and McKell 1988), a few $10 \mu \mathrm{m}$ in pure diamond (Barjon et al 2012). At comparable doping, the spatial extent of diffusion is generally much shorter in direct semiconductors due to faster recombination of excess charge carriers. In the presence of impurities and defects, carrier diffusion lenghts fall in the nanometer range (Gustafsson et al 1998, Barjon et al 2003).

For diffusion lengths which reach values noticeably below the micrometer range, it becomes necessary to use techniques 
with a precise excitation control at the nanometer scale. In the recent years, cathodoluminescence (CL) setups have been fitted to SEM equipped with field emission gun (FEG). They are capable to supply nanometer sized probes at low voltages for the investigation of semiconductor nanostructures (Matsuo et al 1996, Sekiguchi et al 2004, Phillips 2006). This kind of set-ups opens the way to perform direct diffusion measurements by optical and contactless technique with an accuracy of few nanometers.

Since several years, $\mathrm{ZnO}$ has been the subject of intense researches because of its wide band gap energy $(3.3 \mathrm{eV})$ which makes this material interesting for optoelectronic devices emitting in the visible and near UV range (Klingshirn et al 2010). For this purpose, material studies have been undertaken to develop band-gap engineering expertise involving essentially $\mathrm{ZnO} / \mathrm{ZnMgO}$ or $\mathrm{ZnO} / \mathrm{ZnCdO}$ systems to fabricate well defined $2 \mathrm{D}$ or $1 \mathrm{D}$ heterostructures or quantum wells. In this context, the understanding and optimization of the excess charge properties of $\mathrm{ZnO}$ and related alloys are an important issue for the realization of efficient optoelectronic devices.

Even without intentional doping, $\mathrm{ZnO}$ and $\mathrm{ZnMgO}$ crystals generally present a n-type electrical conductivity with high concentrations of free electrons $/ \mathrm{cm}^{3}$ at room temperature. Such a doping favors the exciton population with respect to the free carrier one. In the case of $\mathrm{ZnO}$ with an exciton binding energy of $60 \mathrm{meV}$ and $n=10^{18} \mathrm{~cm}^{-3}$, the mass action law (Im et al 1997) indicates that the exciton density is about one hundred times superior to the free charge carriers at $300 \mathrm{~K}$ in weak excitation regime. That is the reason why, in the following recombination and diffusion processes are considered as governed by excitons.

Few studies have been reported on exciton diffusion lengths in bulk $\mathrm{ZnO}$ and $\mathrm{ZnMgO}$ (Zippel et al 2009, Noltemeyer et al 2012). Values ranging from $75 \mathrm{~nm}$ to $220 \mathrm{~nm}$ were measured depending on the temperature and the content on $\mathrm{Mg}$. For bulk $\mathrm{ZnO}$, the behavior of exciton diffusion with increasing temperature has been interpreted by scattering processes involving deformation and Fröhlich potentials. Lower values are observed for $\mathrm{ZnMgO}$ alloys. On the other hand, exciton diffusion in $\mathrm{ZnO}$-based nanowires has attracted more attention (Yoo and Dang 2008, Hwang et al 2011, Donatini et al 2016a). Beside this, the problematic influence of non-radiative surface recombination was pointed out some time ago (Bylander 1978, Zhao et al 2008). This obviously must be taken into account to correctly interpret experimental diffusion length measurement particularly for the design and optimization of nanostructures-based optoelectronic devices.

In this paper, cathodoluminescence is used to measure the diffusion length of excitons in bulk $\mathrm{ZnMgO}$ at room temperature thanks to specifically designed samples. The effects of non-radiative surface recombination are evidenced with small angle beveled samples prepared using focused ion beam (FIB). It is responsible for a strong shrinking of the diffusion range in nanometer-thick FIB lamella. This kind of thin-lamella preparation, usually dedicated to transmission electron microscopy, have a strong potential to improve the spatial resolution of CL images. (a)

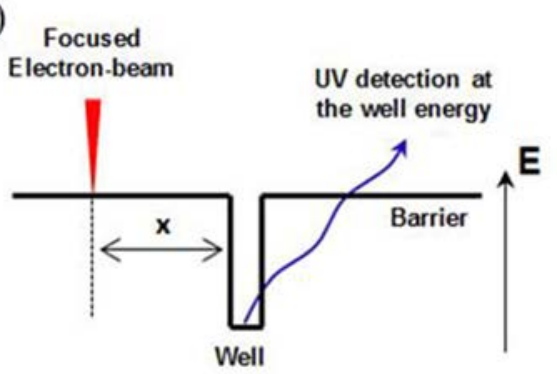

(b)

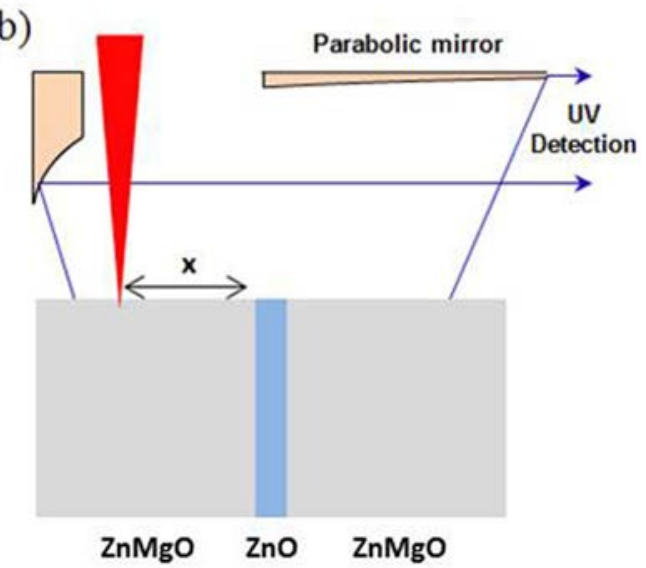

Figure 1. (a) Basic principle of a 1D diffusion experiment. (b) CL experimental configuration (3D): the electron beam is scanned perpendicularly to a single $\mathrm{QW} \mathrm{ZnO} / \mathrm{ZnMgO}$ sample prepared as a cross section.

\section{Method, samples and experimental conditions}

\section{1. $C L$ diffusion experiment}

The basic principle of CL diffusion experiments presented in this work is illustrated at one dimension (1D) in figure 1(a). Like in a pump-probe experiment, excitons are locally generated under an electron beam focused on the barrier at a distance $\mathrm{x}$ (pump) from a collecting well (probe). Excitons diffusing in the barrier are collected in the well where they recombine radiatively. Re-absorption of the light emitted by the well does not occur in the barrier which has larger bandgap energy.

In steady-state regime, the exact solution of the 1D diffusion equation is particularly simple. The density of excitons $n(x)$ collected in the well as a function of the distance $x$ to the well writes $n(x)=n(0) \exp (-x / L)$, where $L$ is the diffusion length of excitons in the barrier material. Assuming that the intensity of radiative recombinations in the well is proportional to its population $n(x)$, the study of $I(x)$ as a function of the distance $x$ to the well provides a measurement of the diffusion length of excitons in the barrier, by using:

$$
I(x)=I(0) \exp (-x / L) .
$$

In practice, the CL diffusion experiments are three dimensional (3D). The experimental configuration used here to measure exciton diffusion by cathodoluminescence is shown in figure 1(b). CL linescan acquisitions are performed on a cross section of the specimen in order to record $I(x)$ along a line perpendicular to the well plane. As soon as the non-radiative 


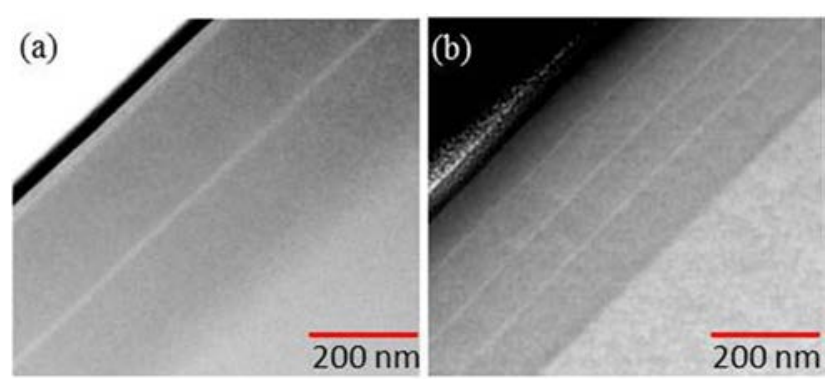

Figure 2. HAADF STEM images: (a) sample $A$ with a single $\mathrm{ZnO}$ QW embedded in $\mathrm{Zn}_{0.92} \mathrm{Mg}_{0.08} \mathrm{O}$ barriers; (b) sample $B$ with three $\mathrm{ZnO}$ QWs. ZnO QWs appear bright and $\mathrm{ZnMgO}$ layers appear darker. The heterostructures were grown on $(11-20)$ non-polar $\mathrm{ZnO}$ substrates.

surface recombination velocity $v_{\mathrm{s}}$ is negligible, the solution of the $3 \mathrm{D}$ diffusion equation in this geometry is the same than (equation (1)) at 1D (Berz and Kuiken 1976). Such an experiment might be used to assess the 3D diffusion length $L_{3 \mathrm{D}}$ occurring in the bulk of the crystal. However, care has to be taken to check the validity of the $v_{\mathrm{s}} \approx 0$ assumption, as seen later.

The geometry of thin lamellas, such as studied in Part 4 with FIB preparation, is almost bidimensionnal (2D). In the case of a collecting line, equation (1) is also valid without any assumption. However the extracted diffusion lengths $L_{2 \mathrm{D}}$ will have a different physical meaning, as discussed in Part (4).

\subsection{Samples and their preparation}

Two samples (referenced $A$ and $B$ ) were homo-epitaxially grown by metal organic vapor phase epitaxy (MOVPE) on $\mathrm{ZnO}$ substrates (supplied by CrysTec $\mathrm{GmbH}$ ) orientated on the $A$-plane $(11-20)$. Sample $A$ consists of a single $\mathrm{ZnO}$ quantum well (QW) embedded in $\mathrm{Zn}_{0.92} \mathrm{Mg}_{0.08} \mathrm{O}$ barriers, while three $\mathrm{ZnO} \mathrm{QW}$ s are embedded in sample $B$. The $A$-plane is non polar, which avoids the appearance of electrical fields along the growth direction causing a quantum confined Stark effect. The choice of non-polar crystals is important for the correct realization of diffusion experiments: the absence of polarization ensures a constant radiative efficiency of the QW upon the collected carrier density.

For the MOVPE process, diethyl-zinc (DEZn), bismethylcyclopentadienyl-magnesium $\quad\left((\mathrm{MCp})_{2} \mathrm{Mg}\right), \quad$ and nitrous oxide $\left(\mathrm{N}_{2} \mathrm{O}\right)$ have been used as zinc, magnesium, and oxygen sources, respectively. Carrier gas was helium. The $\mathrm{ZnO}$ quantum wells and $\mathrm{ZnMgO}$ barriers were deposited at $950{ }^{\circ} \mathrm{C}$ under high $\mathrm{VI} / \mathrm{II}$ mole ratio $\left(\mathrm{R}_{\mathrm{VI} / \mathrm{II}}>6000\right)$ to obtain a bi-dimensional growth and smooth surfaces. Reactor pressure was 50 Torr.

Figure 2 shows scanning transmission electron microscopy (STEM) images performed using a Jeol 2200FS microscope. They were acquired using a high angular annular dark field (HAADF) detector, giving a contrast which is proportional to the material density. As a result, $\mathrm{ZnO}$ appears bright while the lighter $\mathrm{ZnMgO}$ alloy appears dark. The images show that barriers in sample $A$ are $176 \mathrm{~nm}$ thick. In sample $B$, the three QWs are embedded between 70/83/80/110 nm thick barriers. The energy of the excitonic emission from the QW is observed at $3.34 \mathrm{eV}$ (Chauveau et al 2008) at $300 \mathrm{~K}$ which is coherent with a $3 \mathrm{~nm}$ QW thickness. This value is also close to the $5 \mathrm{~nm}$ nominal thickness of the QW estimated from the growth rate. The narrow linewidth $(7 \mathrm{meV})$ of the QW CL recorded at low temperature (see supplementary information figure S1 (stacks.iop.org/JPhysCM/29/485706/ mmedia)) indicates the good quality of the wells. The magnesium composition of the alloyed barriers is measured by energy-dispersive $\mathrm{x}$-ray spectroscopy (EDX) at $8 \%$. Hall effect measurements on a $\mathrm{Zn}_{0.92} \mathrm{Mg}_{0.08} \mathrm{O}$ layer grown in similar conditions on a sapphire substrate shows that majority carriers are free electrons with $n=3.4 \times 10^{17} \mathrm{~cm}^{-3}$ at $300 \mathrm{~K}$. No extended defects such as threading dislocations are observed by STEM, due to a weak lattice mismatch between $\mathrm{ZnO}$ and $\mathrm{Zn}_{0.92} \mathrm{Mg}_{0.08} \mathrm{O}$ epilayers (Kaschner et al 2002) ensuring negligible strain effects. The total thicknesses of the homoepitaxial stackings is $355 \mathrm{~nm}$, almost identical for samples $A$ and $B$.

For diffusion experiments, the samples were prepared as cross sections by two different techniques. The first one is a cleavage along the $c$-plane. This preparation will be used (part 3) to measure the diffusion length of excess charge carriers in the bulk $\mathrm{ZnMgO}$ barrier. The second is the preparation of thin cross sections by the focused ion beam (FIB) technique. It was performed with $30 \mathrm{keV} \mathrm{Ga}$ ions. For the finishing step, the ion voltage was reduced to 5-7 keV to minimize the surface amorphization. With this procedure, thin slabs of sample $A$ and $B$ were prepared in a bevel geometry, with an angle of about 2 degrees. As a result, the sample thickness, labeled $t$, regularly decreases from $300 \mathrm{~nm}$ to $0 \mathrm{~nm}$, as checked with scanning electron microscope (SEM) images. CL linescans were realized for different thicknesses of thin FIB samples. Thanks to contamination marks clearly visible in SEM, it was possible to measure the blade thickness exactly where each linescan CL was performed. Such experiments will be dedicated (Part 4) to probe the effects of surface recombination on exciton diffusion.

\subsection{CL experiments: accurate control of the excitation location with a FEG-SEM}

Samples are excited locally with the focused electron beam of an SEM JEOL $7001 F$ equipped with a field effect gun (FEG). The collection of the radiative exciton recombinations in the QW is realized thanks to a RPM2000 parabolic mirror and analyzed with a Horiba Jobin Yvon optical system equipped with the CL Link e-beam drive unit (developed in collaboration with GEMaC). The excitonic luminescence from the well is spectrally selected using a TRIAX550 monochromator equipped with a silicon CCD camera. CL images are also recorded using a UV photomultiplier Hamamatsu mounted on the second exit port of the monochromator. All the diffusion experiments are performed at $300 \mathrm{~K}$.

The lateral extension $r_{\mathrm{g}}$ of the electron-hole pair generation volume is the relevant parameter to estimate the accuracy of the diffusion-length measurement: 

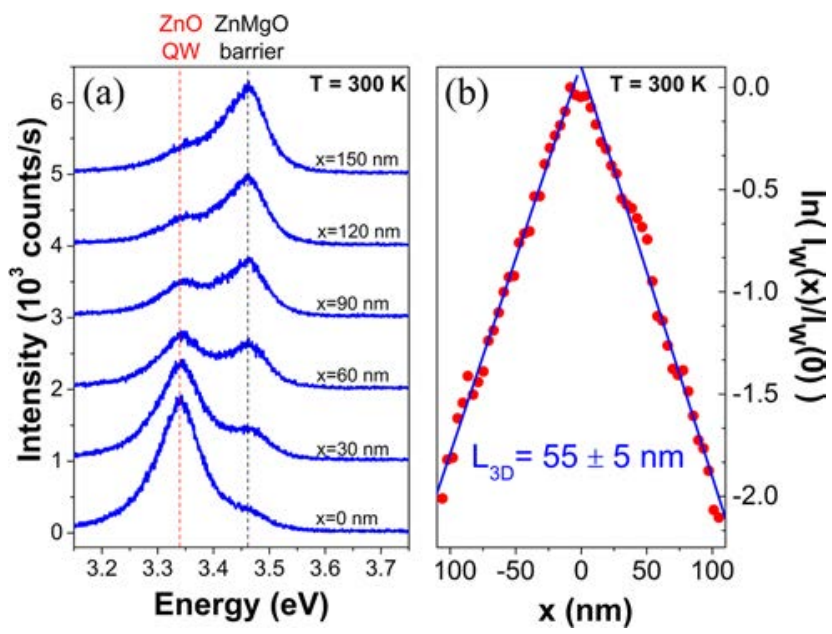

Figure 3. (a) Room-temperature CL spectra collected from the cleaved cross-section when exciting at different distances $x$ from the QW. The spectra are shifted vertically for a better clarity. (b) A linear regression on the logarithmic of $I_{\mathrm{W}}(x) / I_{\mathrm{W}}(0)$ is used to measure $\mathrm{L}$, the diffusion length of excitons in $\mathrm{ZnMgO}$ (details in the text). The step between two electron beam impacts is $\sim 4 \mathrm{~nm}$. The incident electron energy is $E_{0}=2.5 \mathrm{keV}$ and the electron beam current $i=0.1 \mathrm{nA}$.

$$
r_{\mathrm{g}}=\sqrt{\varnothing^{2}+\sigma^{2}}
$$

where $\varnothing$ is the electron beam diameter focused at the specimen surface and $\sigma$ is the lateral spread due to the electron scattering within matter (see supplementary materials figure S2). When using a FEG gun, the electron beam diameter is a few nanometer size. For instance in the conditions used here: $\varnothing \approx 3 \mathrm{~nm}$ at $2.5 \mathrm{keV}$ and $\varnothing \approx 1.5 \mathrm{~nm}$ at $30 \mathrm{keV}$ according to SEM manufacturer specifications.

The best accuracy in CL diffusion experiments are achieved according to different strategies in the cases (i) of the semiinfinite 3D medium formed by a cleaved cross-section or (ii) of the quasi-2D systems formed by FIB thin slabs. Table 1 shows the calculated values of $\sigma$ by using Monte-Carlo simulations (Casino software (Drouin et al 2007)) of electrons accelerated on a bulk $\mathrm{ZnO}$ crystal or on thin slabs of 100 and $20 \mathrm{~nm}$ thicknesses (supplementary materials figure S2). In the bulk case (i), $\sigma$ increases with $E_{0}$, so that low acceleration-voltages are preferred. In case (ii), at high acceleration-voltages the thin lamellae become transparent which strongly reduces $\sigma$. On the basis of table 1 and equation (2), we found $r_{\mathrm{g}}=8.2 \mathrm{~nm}$ at $2.5 \mathrm{keV}$ in bulk $\mathrm{ZnO}, r_{\mathrm{g}}=2.6 \mathrm{~nm}$ in a $100 \mathrm{~nm}$-thick preparation at $30 \mathrm{keV}$ and $r_{\mathrm{g}}=1.5 \mathrm{~nm}$ in a $20 \mathrm{~nm}$-thick preparation at $30 \mathrm{keV}$. These values indicate the spatial resolution of the diffusion length measurements with a FEG-SEM, hereafter.

\section{Bulk diffusion length of excitons in ZnMgO ternary alloy}

\subsection{Experiment on the cleaved cross-section (sample A)}

In figure 3(a) CL spectra from the cleaved cross section of sample $A$ are reported for different distances $x$ from the QW. For an excitation located far from the QW, the barrier
Table 1. Lateral spread $\sigma$ of the electron beam in $\mathrm{ZnO}$ calculated by Monte-Carlo simulations (see conditions in the supplementary information figure $\mathrm{S} 2$ ).

\begin{tabular}{lll}
\hline $\mathrm{ZnO}$ sample & $\sigma(2.5 \mathrm{keV})$ & $\sigma(30 \mathrm{keV})$ \\
\hline Bulk & $7.7 \mathrm{~nm}$ & $550 \mathrm{~nm}$ \\
$100 \mathrm{~nm}$ thick & $7.7 \mathrm{~nm}$ & $1.9 \mathrm{~nm}$ \\
$20 \mathrm{~nm}$ thick & $0.9 \mathrm{~nm}$ & $<0.1 \mathrm{~nm}$ \\
\hline
\end{tabular}

luminescence predominates in the spectra with a maximum at $3.46 \mathrm{eV}$. The $\mathrm{CL}$ intensity emitted from the $\mathrm{ZnO} \mathrm{QW}$ appears only very weak at $3.34 \mathrm{eV}$. When the distance $\mathrm{x}$ between the generation region and the $\mathrm{QW}$ decreases, the light emission intensities simultaneously increase for the QW and decrease for the $\mathrm{ZnMgO}$ barrier. These observations highlight the efficient transfer of excitons from the $\mathrm{ZnMgO}$ barrier to the $\mathrm{ZnO}$ QW. At $x=0$, the QW CL intensity reaches a maximum, since the incident electron beam directly impacts the QW.

In figure $3(\mathrm{~b}) \ln \left(I_{\mathrm{W}}(x) / I_{\mathrm{w}}(0)\right)$ is plotted as a function of $x$. The data plot is clearly linear and is well symmetrical in the upper and lower $\mathrm{ZnMgO}$ layers. According to equation (2), a linear regression on such a plot gives the diffusion length of excitons in the barrier. We found $L=55 \pm 5 \mathrm{~nm}$ in the $\mathrm{ZnMgO}$ barrier at room temperature along a-direction. Note that in principle this diffusion length should be considered as an effective diffusion length since it takes into account the recombination velocity at the cleaved surface. However, the observation of a linear behavior over 2 decades indicates that the effective diffusion length of $55 \mathrm{~nm}$ is very close to the bulk value (Donolato 1982). This will be further confirmed in Part 4.

The result of the diffusion experiment is consistent with the order of magnitude of $75 \mathrm{~nm}$ reported by Zippel et al (2009) for $\mathrm{ZnO} / \mathrm{Zn}_{0.83} \mathrm{Mg}_{0.17} \mathrm{O}$ heterostructures grown by pulsed laser deposition. The diffusion length in the $\mathrm{ZnMgO}$ ternary alloy is found lower than the $110 \mathrm{~nm}$ value reported in bulk $\mathrm{ZnO}$ (Noltemeyer et al 2012). This is consistent with a general trend to observe a lower extent of the carrier diffusion in a ternary than in binary semiconductor compounds (Gustafson 1998). First, the growth conditions of ternary alloys are more difficult to control so that they generally present a higher density of defects compared to binary compounds. Second, the scattering of carriers on the potential fluctuations-statistical or due to composition inhomogeneities-probably also contributes to limit the extent of exciton diffusion in ternary alloys.

\subsection{Luminescence efficiency of $Q W$ heterostructures}

To check the consistency of previous diffusion results, CL experiments were also performed as a function of the incident electron beam energy in the more conventional configuration shown in inset of figure 4: Incident electrons are oriented along the growth axis and impact the as-grown surface. The beam current $i$ was adjusted in order to keep a constant $0.6 \mu \mathrm{W}$ excitation power $\left(P=i E_{0}\right)$. Figure 4 then provides a comparison of sample $A$ and $B$ luminescence yields, with either 1 or 3 QWs. The CL of a bulk $\mathrm{ZnO}$ single crystal (CrysTec substrate) recorded in the same conditions is also shown for comparison. 


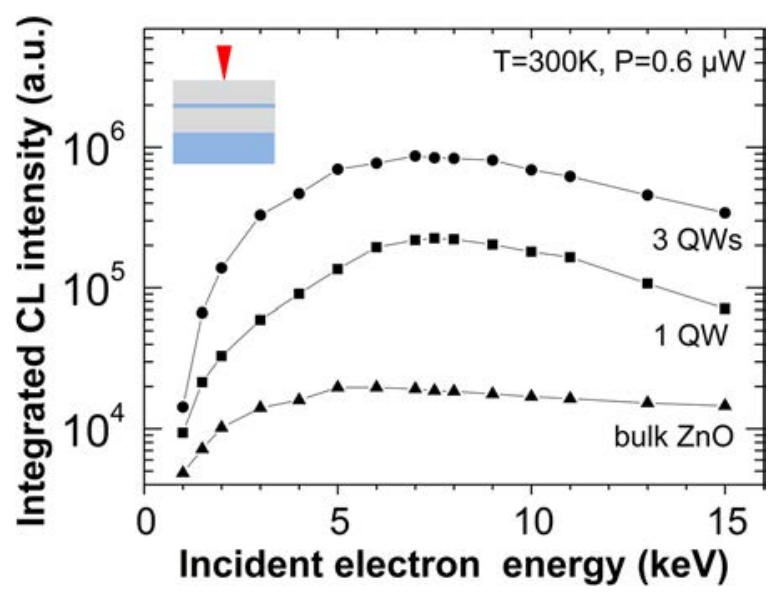

Figure 4. Integrated CL intensity emitted as a function of the incident electron energy $E_{0}$. The excitation geometry is shown in inset and a bulk $\mathrm{ZnO}$ sample is presented for comparison. The incident power $P=i E_{0}$ was kept to a constant value of $0.6 \mu \mathrm{W}$.

In all samples, the CL intensity drops at low voltage. This is an effect of non-radiative recombinations occurring at the sample surface. Indeed, the lower the incident electron energy, the closer the carriers are injected from the surface and the higher the luminescence losses by non-radiative surface recombinations. The maximum CL yield is observed for electrons of about 7-8 keV incident energy in $\mathrm{ZnO} \mathrm{QW}$ samples. At higher energies, the CL emission decreases again because excitons are generated deeper, directly in the $\mathrm{ZnO}$ substrate rather than in the $\mathrm{ZnMgO} / \mathrm{ZnO} / \mathrm{ZnMgO}$ epitaxial layers. In the bulk $\mathrm{ZnO}$ substrate, excitons recombine with a much lower radiative efficiency than in a $\mathrm{QW}$. The maxima of light emissions occur at $7-8 \mathrm{keV}$ for both the single- and the three-QW heterostructures simply because the QWs are centered in the multilayers which have comparable thicknesses.

More interestingly, the CL yield is almost 4 times higher with $3 \mathrm{QWs}$ than with a single QW. To interpret this result with respect to diffusion experiments presented previously, one has to consider that most of excitons are generated in the $\mathrm{ZnMgO}$ barrier rather than in the QW. Indeed, the direct excitation of QWs by energy losses of incident electrons is extremely weak. As a quantitative illustration, the QW is excited by only $3 \%$ of the deposited energy in sample $A$ at $8 \mathrm{keV}$. In fact, the $\mathrm{CL}$ yield experiments of figure 4 reveal the ability of $\mathrm{ZnO}$ QWs to collect excitons diffusing in the $\mathrm{ZnMgO}$ layers. In sample $A$ having a single $\mathrm{QW}$, the diffusion length in $\mathrm{ZnMgO}$ ( $L_{3 \mathrm{D}}=55 \mathrm{~nm}$ measured previously) is much shorter than the $\mathrm{ZnMgO}$ barrier thickness $(175 \mathrm{~nm})$, hence a large amount of excitons are lost. On the contrary in sample $B$, the diffusion length is comparable to the thicknesses of $\mathrm{ZnMgO}$ layers $(70 / 83 / 80 / 110 \mathrm{~nm})$. The 4 times higher luminescence yield of sample $B$ is explained by a more efficient capture of excitons in the three QWs. As a summary, the CL yield of the samples is consistent with the $55 \mathrm{~nm}$ diffusion length found for bulk $\mathrm{ZnMgO}$.

\section{Surface recombinations in thin slabs prepared by FIB}

In this part, the exciton diffusion is investigated in the quasi2D geometry of thin beveled slabs prepared by FIB, as illustrated in figure 5(b). Linescans were performed across the single QW of sample $A$ for different slab thicknesses $t$. Figure 5(a) shows the corresponding logarithmic plots. Again the $\ln \left(I_{\mathrm{w}}(x) / I_{\mathrm{w}}(0)\right)$ plots present an almost linear form and effective diffusion lengths are deduced.

Figure 5(c) displays the dependency of the effective diffusion length in $\mathrm{ZnMgO}$ with the thicknesses $\mathrm{t}$ of the FIB preparation. The effective diffusion length in the thicker part $(t \sim 260 \mathrm{~nm})$ of the FIB bevel is $44 \pm 12 \mathrm{~nm}$. This result is consistent with the $55 \mathrm{~nm}$ diffusion length measured before from the cleaved cross section. A dramatic shrinkage of the diffusion extent is observed in the thinnest parts. In the thinnest regions of the FIB bevel $(t \sim 30 \mathrm{~nm})$, the effective diffusion length decreases down to $8 \pm 2 \mathrm{~nm}$. With high surface to volume ratios $(S / V)$ in the thinnest part of the nanometric bevel, the influence of non-radiative surface recombinations is qualitatively expected to play a much more important role.

Quantitatively, the bulk diffusion length $L_{3 \mathrm{D}}$ of injected carriers in $\mathrm{ZnMgO}$ has been assessed in Part 3 as $L_{3 \mathrm{D}}=\sqrt{D \tau_{3 \mathrm{D}}}$. In the 2D geometry of FIB lamellas investigated here, the effective diffusion lengths write $L_{2 \mathrm{D}}=\sqrt{D \tau_{2 \mathrm{D}}}$, where

$$
\frac{1}{\tau_{2 \mathrm{D}}}=\frac{1}{\tau_{3 \mathrm{D}}}+\frac{1}{\tau_{\mathrm{s}}} .
$$

In this expression $\tau_{3 \mathrm{D}}$ is the lifetime of excitons in a bulk $\mathrm{ZnMgO}$ crystal and $\tau_{\mathrm{s}}$ is the exciton lifetime at surface. For thin layers, the latter expresses with the surface recombination velocity (Langer and Walukiewicz 1995) $v_{\mathrm{s}}$ as $1 / \tau_{\mathrm{s}}=2 v_{\mathrm{s}} / t$. The following expression is used as a first approach for describing the experimental results:

$$
L_{2 \mathrm{D}}=L_{3 \mathrm{D}}\left(1+\frac{2 \tau_{3 \mathrm{D}} v_{\mathrm{s}}}{t}\right)^{-1 / 2} .
$$

For thin preparations, it indicates that the effective diffusion length is almost proportional to the square root of the preparation thickness. Given a bulk exciton lifetime in $\mathrm{Zn}_{0.92} \mathrm{Mg}_{0.08} \mathrm{O}$ layers $\tau_{3 \mathrm{D}}=0.4 \mathrm{~ns}$ as measured by timeresolved cathodoluminescence (Donatini et al 2016b), $v_{\mathrm{s}}$ remains the only unknown parameter in equation (4). Our experimental results are reasonably well described by equation (4) with $v_{\mathrm{s}}=7 \times 10^{4} \mathrm{~cm} \mathrm{~s}^{-1}$ at the surface of $\mathrm{ZnMgO}$ (dashed line in figure 5(c)). Though the model deserves to be refined, this value is coherent with previous reports for $v_{\mathrm{s}}$ in $\mathrm{ZnO}$ found in the $1.5-4.5 \times 10^{5} \mathrm{~cm} \mathrm{~s}^{-1}$ range (Bylander 1978, Zhao et al 2008, Donatini et al 2016a). Surface defects induced by the ion bombardment during the FIB preparation might explain the higher recombination velocity observed here.

Finally, we found a reduced surface velocity $S=v_{\mathrm{s}} \tau_{3 \mathrm{D}} / L_{3 \mathrm{D}}$ equal to about 5. This low $S$ value means that the deviation 

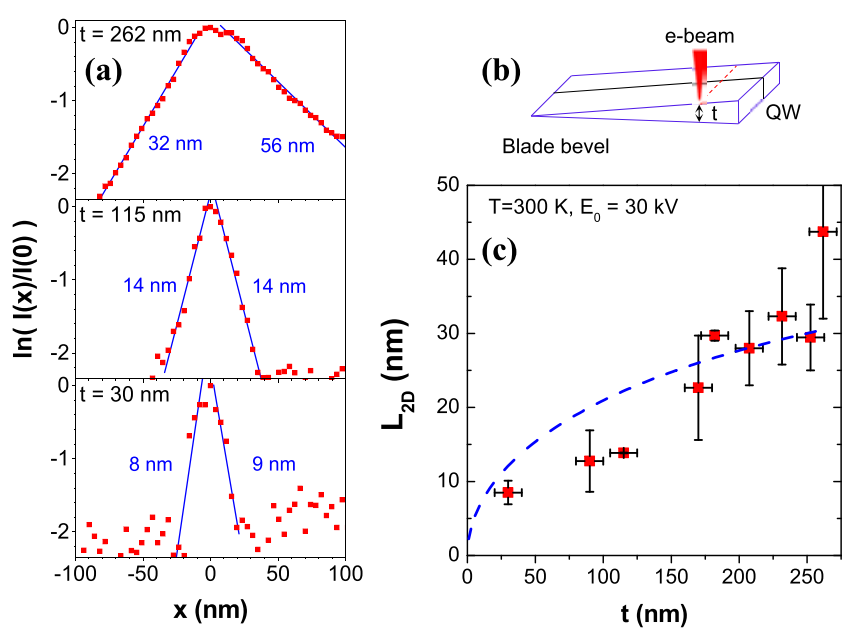

Figure 5. (a) CL linescans taken at different thicknesses, $t$, of the thin lamella prepared by FIB (sample $A$ ), substrate on the right. (b) Scheme of the corresponding experiment. (c) Effective diffusion length $L_{2 \mathrm{D}}$ of excitons in $\mathrm{ZnMgO}$ as a function of the lamella thickness, $t$. Experiments done à $300 \mathrm{~K}$ with $E_{0}=30 \mathrm{keV}$ and $i=0.1 \mathrm{nA}$. The diffusion length values are averaged from both sides of the QW. The dashed curve is a plot of equation (4) with $v_{\mathrm{s}}=7 \times 10^{4} \mathrm{~cm} \mathrm{~s}^{-1}$ (details in the text).

from equation (2) due to surface recombinations is weak for the cleaved cross section investigated in Part 3. This confirms that the measurement $L_{3 \mathrm{D}}=55 \mathrm{~nm}$ is valid for excitons diffusing in the bulk of a $\mathrm{ZnMgO}$ crystal. An uncertainty below $30 \%$ can be further deduced from (Donolato 1982).

\section{Application to high resolution $\mathrm{CL}$ images}

There is a practical interest in using thin cross-section preparations for CL imaging. Actually, the spatial resolution of CL images is most often limited by the diffusion of excess charge carriers (or excitons in the present case). Indeed, the CL images are the combined result of excitation, diffusion and recombination processes. The luminescence signal obtained with a point-source excitation accounts for the spatial distribution of excess carriers by diffusion around the point-source. In other words, CL images might be seen as 'excitation images' meaning that they reveal the spatial distribution of injected excess carriers.

An expression $\sqrt{r_{\mathrm{g}}^{2}+4 L^{2}}$, where $L$ is their effective diffusion length, was proposed in early times (Davidson 1977) for the CL image resolution. It has to be noted that it is not appropriate as a general description of the complex 3D diffusion and recombination problem leading to the formation of CL images (Donolato 1994). However, in the case of CL linescans and images of figure 6, the spatial resolution is limited by the diffusion length of excess carriers, as discussed in what follows.

CL linescans were performed on the slab bevel prepared from sample $B$ with 3 QWs. Figure 6 compares the results obtained from the thick $(\sim 200 \mathrm{~nm})$ and the thin $(\sim 20 \mathrm{~nm})$ regions of the FIB preparation. For the thicker part (figure 6(a)) there is almost no variation of the luminescence intensity from the $\mathrm{ZnO}$

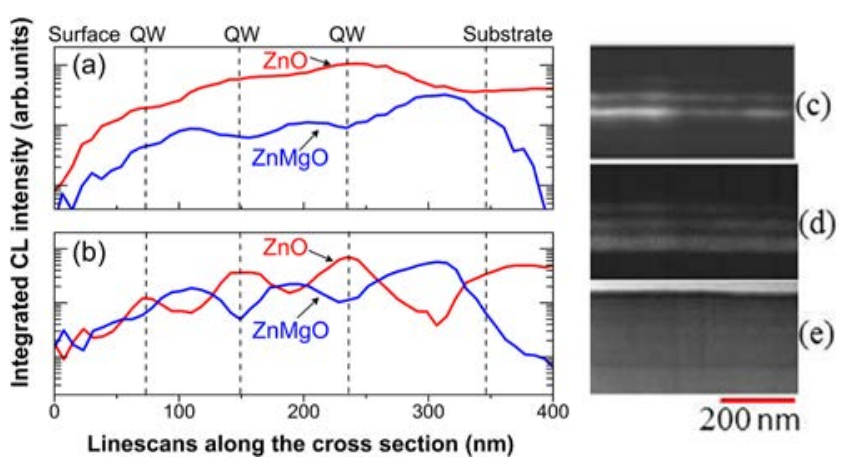

Figure 6. CL linescans along the cross section of sample $B$ prepared by FIB: (a) thick region $t=200 \mathrm{~nm}$, (b) thin region $t=20 \mathrm{~nm}$. CL images obtained in the thin region when filtered at the luminescence energy of: (c) the $\mathrm{ZnO} \mathrm{QW}$ and (d) the $\mathrm{ZnMgO}$ barrier (e) is a secondary electron image simultaneously recorded with the CL images.

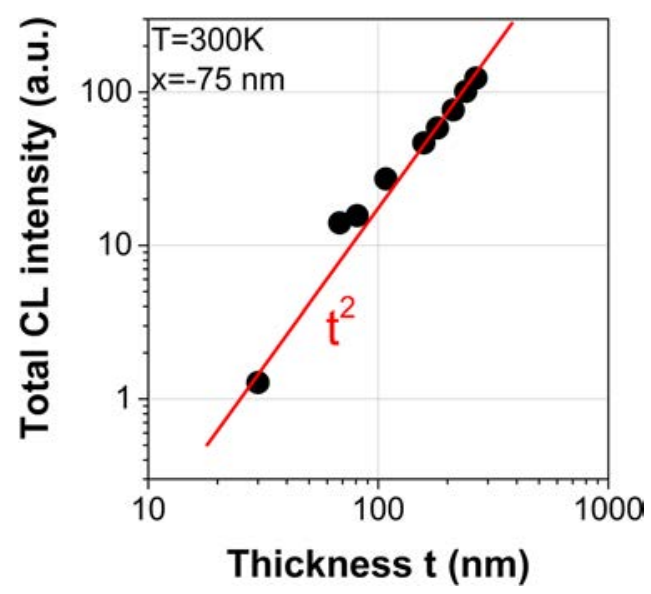

Figure 7. Total CL intensity as a function of the FIB preparation thickness recorded at $x=-75 \mathrm{~nm}$ from the QW. The linear fit (red line) indicates that the CL intensity $I_{\mathrm{CL}} \propto t^{2}$, where $t$ is the thickness of the FIB cross-section.

QWs. In other words, the QW emissions cannot be spatially separated because the distance between QWs is comparable to the carrier diffusion length in the $\mathrm{ZnMgO}$ barrier. On the contrary, in the thinner part (figure 6(b)) where the diffusion length is strongly reduced by surface recombinations, the $\mathrm{ZnO}$ signal exhibits strong spatial variations, which are fully correlated with opposite ones from the $\mathrm{ZnMgO}$ signal. Consequently, the identification of each $\mathrm{QW}$ becomes possible. This result is further illustrated in figures 6(c) and (d) where CL images from the thinnest part of the FIB lamella are strongly contrasted. The ZnO QW emissions are well spatially separated. These experiments highlight that, with thin FIB cross sections, the decrease of exciton diffusion due to surface effects significantly improves the spatial resolution of CL images.

However, the improvement of the spatial resolution in CL images has a cost. The counterpart of the diffusion length reduction is a strong $\mathrm{CL}$ signal decrease, which is evidenced in figure 7 as a function of the slab thickness. At $E_{0}=30 \mathrm{keV}$, most of electrons are transmitted through the thin lamella (transparency regime). Then the energy absorbed 
by the lamella is almost proportional to its thickness. In other words, the electron-hole generation rate $g$ is proportional to $t$. As $\tau_{2 \mathrm{D}} \approx 2 v_{\mathrm{s}} / t$ in the thin lamella limit, the CL intensity $I=g\left(\tau_{2 \mathrm{D}} / \tau_{\text {rad }}\right)$ is almost proportional to $t^{2}$ (Yuan et al 1989). In figure 7 we observe a good agreement with this simple description, showing that the effective diffusion length reduction is accompanied by a quadratic decrease of the luminescence efficiency. At the end, the improvement for the CL resolution with thin preparations appears as a matter of compromise between signal and resolution.

\section{Conclusions}

In this work, the diffusion of excitons injected in $\mathrm{ZnMgO}$ is investigated at the room temperature thanks to $\mathrm{ZnO} / \mathrm{ZnMgO}$ non polar heterostructures. First, a diffusion length of $55 \mathrm{~nm}$ for excitons in $\mathrm{ZnMgO}$ is measured by CL linescans on a cleaved cross section. This value corresponds to the bulk diffusion length in the $\mathrm{ZnMgO}$ ternary alloy. It well explains the improved collection of excitons observed in multi-QW, attested by their higher luminescence yield compared with single QW structures. In the future, heterostructures based on $\mathrm{ZnCdO} / \mathrm{ZnO}$ quantum wells appear promising to extend the proposed approach for the investigation of exciton diffusion in $\mathrm{ZnO}$.

The effects of dimension reduction $(3 \mathrm{D} \rightarrow 2 \mathrm{D})$ on exciton diffusion have been further studied with thin slabs prepared by FIB from the same samples. It is observed that the effective diffusion length is significantly reduced by non-radiative surface recombinations. Incidentally, thin preparations can be exploited to improve the spatial resolution of CL images in a compromise with the luminescence intensity reduction. A first order approach has been proposed to evaluate the recombination velocity, giving coherent values with published data. The model could be refined by a theoretical description of diffusion processes in the case of high incident electron energies, where a uniform generation line crosses electron-transparent samples, which has not been reported up to now.

\section{Acknowledgments}

The authors would like to thank D Troadec from IEMN, Villeneuve d'Ascq, France for the FIB bevel preparations and their thickness measurements. The authors are grateful to $\mathrm{C}$ Vilar for her precious technical help on the SEM-CL set-up, to E Chikoidze for Hall effect measurements and to M Phillips for stimulating discussions and to J Chevallier for his critical reading of the manuscript.

\section{References}

Barjon J, Jomard F, Tallaire A, Achard J and Silva F 2012 Appl. Phys. Lett. 100122107

Barjon J, Brault J, Daudin B and Jalabert D 2003 J. Appl. Phys. $942755-7$

Berz F and Kuiken H K 1976 Solid-State Electron. 19 437-45

Bylander E G 1978 J. Appl. Phys. 49 1188-95

Chauveau J-M, Laügt M, Venneguès P, Teisseire M, Lo B, Deparis C, Morhain C and Vinter B 2008 Semicond. Sci. Technol. 23035005

Davidson S M 1977 J. Microsc. 110 177-204

Donatini F, Luna de Bugallo A, Tchoulfian P, Chicot G, Sartel C, Sallet V and Pernot J 2016a Nanoletters 16 2938-44

Donatini F, Arnold C and Barjon J 2016b private communication

Donolato C 1994 Phys. Status Solidi a 14 K131-32

Donolato C 1982 Solid-State Electron. 25 1077-81

Drouin D, Couture A R, Joly D, Tastet X, Aimez V and Gauvin R 2007 Scanning 29 92-101

Gustafsson A, Pistol M-E, Montelius L and Samuelson L 1998 J. Appl. Phys. 84 1715-75

Hwang J-S, Donatini F, Pernot J, Thierry R, Ferret P and Dang L S 2011 Nanotechnology 22475704

Im J S, Moritz A, Steuber F, Härle V, Scholz F and Hangleiter A 1997 Appl. Phys. Lett. 70 631-3

Kaschner A et al 2002 Appl. Phys. Lett. 80 1909-11

Klingshirn C F, Meyer B K, Waag A, Hoffmann A and Geurts J 2010 Zinc Oxide From Fundamental Properties Towards Novel Applications (Springer Series in Materials Science) (Berlin: Springer)

Langer J M and Walukiewicz W 1995 Mater. Sci. Forum 196-201 1389

Matsuo H, Kobayashi N, Kimura Y and Shimizu R 1996 J. Electron Microsc. 45 453-57

Noltemeyer M, Bertram F, Hempel T, Bastek B, Polyakov A, Christen J, Brandt M, Lorenz M and Grundmann M 2012 J. Mater. Res. 27 2225-31

Phillips M R 2006 Mikrochim. Acta 155 51-8

Saritas M and McKell H D 1988 J. Appl. Phys. 63 4561-7

Sekiguchi T, Liu Q, Tanaka T, Hu J, Zhu Y and Bando Y 2004 Eur. Phys. J. Appl. Phys. 27 107-9

Yoo J, Yi G-C and Dang L S 2008 Small 4 467-70

Yuan J, Berger S D and Brown L M 1989 J. Phys.: Condens. Matter $13253-65$

Zhao Q X, Yang L L, Willander M, Sernelius B E and Holtz P O 2008 J. Appl. Phys. 104073526

Zippel J, Lenzner J, Benndorf G, Lange M, Hochmuth H, Lorenz M and Grundmann M 2009 J. Vac. Sci. Technol. B 27 1735-40 